\title{
Travel times, rational queueing and the macroscopic fundamental diagram of traffic flow
}

\author{
Dieter Fiems $^{a}$, Balakrishna Prabhu ${ }^{b}$ and Koen De Turck ${ }^{c}$ \\ ${ }^{a}$ Ghent University, Department TELIN, Belgium \\ ${ }^{b}$ LAAS-CNRS, Université de Toulouse, CNRS, Toulouse, France \\ ${ }^{c}$ CentraleSupélec, Paris, France
}

November 21, 2018

\begin{abstract}
We propose a Markovian queueing model for computing travel times at a macroscopic scale during rush hour. The service rates of the queueing model are state-dependent, reflecting the speed/density relation of the fundamental diagram of traffic flow. In the fluid limit, the dynamics of the transient queue size and travel time processes are governed by a set of differential equations. As an application of the model, we consider the rational time-dependent choice between public and private transport, assuming that there is a congestion-free public alternative to private transportation. Numerical examples reveal that a small reduction in peak traffic can significantly reduce the average travel times.
\end{abstract}

\section{Introduction}

"How long will it take?" is one of the first questions that is asked before one goes on a journey. This question is often accompanied by apprehension for journeys through urban areas during rush hour when the risk of congestion and grid-locks is high. Can we save ourselves the anxiety by estimating average travel times just like we do with weather bulletins? This is one of the main questions that we seek to answer affirmatively in this paper.

The estimates for the travel times will be derived using concepts from queueing theory [1]. The analogy between customers in queues and cars on roads is a natural one that has been brought up in the modelling of various phenomena in road traffic networks $2[4]$. The computation of the travel times will be based on two factors that have a strong influence on the time spent in a queue: (i) the arrival rate of customers (vehicles, in our case); and (ii) the speed with which the queue can be drained. The statistics of the number of vehicles arriving per hour can be obtained from measurements at various locations in a city (see, for example, $[5$ ). The second factor, which is the average departure rate of vehicles, can be computed at two different spatial scales. At the microscopic level of a road segment, the relationship between the density of vehicles on a road segment and the average rate of departure of vehicles is given by what is known as the fundamental diagram in the transportation theory literature 6 10.

At a coarser scale, Daganzo 11] proposed a macroscopic fundamental diagram that relates the total outflow (vehicles per unit time) from a neighbourhood to the aggregate accumulation (vehicles per unit length of road) for a single-lane system. Figure 1 shows two macroscopic fundamental diagrams from literature, which were obtained from actual measurements in Yokohama $[12$ and Toulouse [13. The macroscopic fundamental diagrams will serve as the departure rate curves for the computation of journey times in our queueing model. 


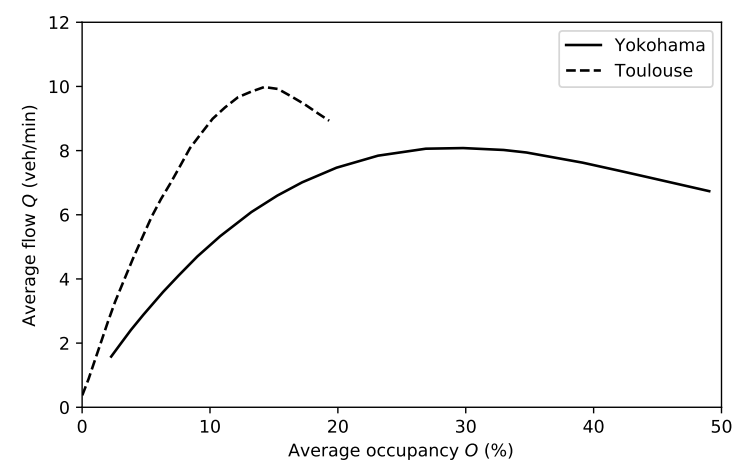

Figure 1: Macroscopic fundamental diagram

Starting from the arrival rate statistics and the macroscopic fundamental diagram of a given area, we give a recipe based on queueing theory to derive the average journey time through that area at any given time of the day. The aim of this study is two-fold: (i) estimation of the journey times per $s e$; and (ii) prediction of the influence of public transport in reducing congestion. The first aim has an inherent interest, for example, to plan at what time to leave in order to arrive at a certain hour at the destination. The second aim is motivated by the park and ride schemes introduced by many cities. In these schemes, commuters can park their vehicles at a public transport hub (usually on the outskirts of a city) and then hop onto the public transport network to arrive at their destination inside the city. The journey times by public transport are usually easy to estimate, especially for metros and trams with dedicated lanes. Bus services too are now being provided with dedicated corridors to reduce the influence of traffic jams and to make the commuting times more predictable. If commuters could also obtain an estimate on how long it would take them to drive to their destination instead of taking public transport, then they could choose to switch to public transport if there is time to be gained. Assuming commuters are rational and seek to minimise their travel time, how many of them will choose to switch to public transport? What influence will this have on the congestion on the roads? The answers to these questions can provide guidelines for dimensioning public transport systems especially at peak hours.

Road traffic models have been investigated from different perspectives, including queueing theory [14, transportation research [15 17, and statistical physics [18 20]. Most queueing models divide a road into a number of sections and model each section as a queueing system, such that a stretch of road constitutes a queueing network [14]. Queueing models may be either stationary [21, in which case the traffic conditions do not change over time or non-stationary (transient) 22 when they do. In both cases, these queueing models are explanatory for the fundamental diagram of traffic theory. In transportation research, cell transmission models [15 17] also divide the road network into sections (cells), and describe the evolution of the number of cars in the cells over time. While in queueing theory, each vehicle spends some random time in a cell, before moving to the next, the transition is deterministic in cell transmission models. In particular, the fundamental diagram is explained by noting that the traffic is constrained by the free space in the next cell, the number of cars in the preceding cell, and a bound on the flow from one cell to the next. The road traffic models from the statistical physics community can be roughly classified in hydrodynamic 23 26], gas-kinetic [27 29], car-following [30 33, and cellular automata 34 38 models. They can be used to study the formation of traffic jams [39.40] and traffic waves [41], for routing traffic based on real-time information 42, or for optimising traffic signals 43 . An in-depth treatment on these and many other models at both microscopic and macroscopic levels can be found in 8, 28, 44, 45. Some traffic models have been combined with game-theoretic concepts to understand the influence of individual decision making. Examples include the computation of the price of anarchy in routing games [46 47, the study of the 


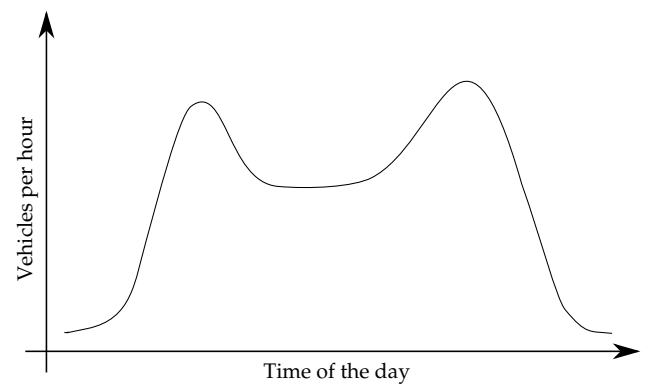

Figure 2: A two-way arrival rate curve (see, e.g., 5] which has statistics from the Vancouver area in Canada)

dilemma of switching lanes 48, 49, or the explanation of Braess' paradox [50]. In a spirit similar to that of statistical physics, models based on fluid queues have also been proposed for the dynamics of traffic flow in discrete time [51] and in continuous time [52,53.

Most of these references, including the literature on queueing and cell transmission models, are concerned with flows on fixed-length segments, and do not deal with the distribution of the journey times on the scale of cities or urban areas with the exception of [54] in which bounds have been obtained for two segments. Further, these works also assume First-in-First-Out (FIFO) traffic which is more suited for segments or routes in which the traffic dynamics after the departure time do not influence the journey times. In our model, vehicles arrive according to a Poisson process and have exponentially distributed journey lengths (distance). Different vehicles usually have different destinations within an urban area and it is natural assume that vehicles have different travel distances and that a vehicle that arrives later can leave earlier.

A problem related to ours that has been investigated by the transportation research community is the so-called morning commute problem. Assuming that drivers have to arrive at their common destination on or before their chosen time, the morning commute problem seeks to determine the departure times of the vehicles in order to minimise the waiting time at the destination. One can seek a centralised solution in which a central planner decides the departure times in order to minimise the total cost or a decentralised solution in which each vehicles chooses its departure time with the aim of minimising its own cost. Initiated by Vickery [51, this problem has been investigated in various settings: 55 assumes a hydrodynamic model and a single route, 56 looks at multiple routes as well as at the impact of tolls, and more recently, 57] adds transit systems to the mix while [58] includes ridesharing systems in their model. These papers again are based on assumptions of fixed segment-lengths and FIFO traffic.

\section{Travel time analysis}

Consider a neighbourhood of a city, the size of which depends upon the scale at which journey times need to be computed, and for which the arrival rate statistics and the macroscopic fundamental diagram are known. The neighbourhood could, in theory, be the entire city itself. We shall assume that new vehicles arrive in accordance to a Poisson process with time dependent rate $\lambda(t)$, where this rate typically has a shape as in Figure 2 .

The duration of a journey depends on the length of the journey as well as the speed during the journey. We assume that the lengths are independently and identically exponentially distributed with rate 1 , taking some other value being equivalent to rescaling time. The speed of vehicles inside this area at a given time instant will depend upon the average density within the area in accordance with the macroscopic fundamental diagram (MFD). Moreover, the number of vehicles that either leave the area or reach their destination will also depend on the density, or equivalently, on the number of vehicles 
in the area.

Let $Q(t)$ denote the number of vehicles (moving around) in the area at time $t$ and let $\pi_{n}(t)=$ $\mathrm{P}[Q(t)=n]$ denote the probability that there are $n$ vehicles in the area at time $t$. Let $D(t)$ be the density of vehicles at time $t$, and let $F(D(t))$ be the average flow when the density is $D(t)$; the function $F$ is obtained from the MFD.

Measurements have shown that the departure rate, $\mu$, (by leaving the area or by reaching the destination) is proportional to the average flow: $\mu \propto F(D(t))$, see 59. Since the traffic density is proportional to the number of vehicles in the area, $D(t) \propto Q(t)$, we obtain the following relation between the departure rate and the number of vehicles:

$$
\mu_{Q(t)}=C F(c Q(t)),
$$

with $c$ and $C$ appropriate proportionality constants.

\subsection{Markov model}

With the above assumptions, $Q(t)$ constitutes a Markov chain with state space $\mathbb{N}$. When there are $Q(t)=n$ vehicles in the area at time $t$, there is an arrival in $(t, t+d t]$ with probability $\lambda(t) d t+o(d t)$, and a departure with probability $\mu_{n} d t+o(d t)$. Hence, the Kolmogorov backward equations read:

$$
\dot{\pi}_{n}(t)=\lambda(t) \pi_{n-1}(t)+\mu_{n+1} \pi_{n+1}(t)-\left(\lambda(t)+\mu_{n}\right) \pi_{n}(t)
$$

for $n \in \mathbb{N}^{+}$, and

$$
\dot{\pi}_{0}(t)=\mu_{1} \pi_{1}(t)-\lambda(t) \pi_{0}(t) .
$$

Let $W(\tau)$ denote the remaining travel time of a vehicle in the area at time $\tau$. Since the arrival rate of vehicles depends on time, the travel time of a vehicle will not be stationary. Let $w_{n}(\tau, t)=\mathrm{P}[W(\tau)>$ $t \mid Q(\tau)=n$ ] denote the complementary distribution function of the remaining travel time $W(\tau)$ of a vehicle in the area, conditioned on having $Q(\tau)=n$ vehicles in the area at time $\tau$. By conditioning on the number of vehicles at time $\tau+h$, we can relate the remaining travel time of vehicles at time $\tau$ and $\tau+h$. We obtain the following relation:

$$
\begin{aligned}
w_{n}(\tau, t+h)=w_{n+1}(\tau+h, t) \lambda(t) h+w_{n}(\tau+h, t)(1-\lambda(t) h & \left.-\mu_{n} h\right) \\
& +w_{n-1}(\tau+h, t) \mu_{n} h\left(1-\frac{1}{n}\right)+o(h),
\end{aligned}
$$

for $n \in \mathbb{N}^{+}$. Sending $h \rightarrow 0$, we further have

$$
\frac{\partial}{\partial t} w_{n}(\tau, t)-\frac{\partial}{\partial \tau} w_{n}(\tau, t)=w_{n+1}(\tau, t) \lambda(t)-w_{n}(\tau, t)\left(\lambda(t)+\mu_{n}\right)+w_{n-1}(\tau, t) \mu_{n} \frac{n-1}{n},
$$

for $n \in \mathbb{N}^{+}$. Finally, the expected travel time of a vehicle that arrives at time $\tau$ is

$$
\bar{W}(\tau)=\sum_{n=0}^{\infty} \pi_{n}(t) \int_{0}^{\infty} w_{n+1}(\tau, t) d t,
$$

where we used the observation that the mean travel time of a vehicle arriving at time $\tau$ while there are $n$ vehicles in the system equals the mean remaining travel time of a vehicle when there are $n+1$ vehicles in the area.

\section{$2.2 \quad$ Fluid limit}

As the fundamental diagram translates to state-dependent service rates, there is no simple analytic solution for the infinite systems of differential equations for $\pi_{n}(t)$ and $w_{n}(\tau, t)$. Moreover, numerically 
solving these equations is computationally demanding. We however can study the fluid limit of the system, that is, we consider a sequence of models $Q^{N}(t)$, the $N$ th model having arrival rate $\lambda(t) N$ and study $q(t)=\lim _{N \rightarrow \infty} Q^{N}(t) N^{-1}$. The parameter $N$ is the scale at which we are making measurement. As the scale becomes large, the number of vehicles also grows proportionally, and hence we have to divide $Q$ by $N$ to obtain a quantity which goes to a finite limit. This quantity can be thought of as the density of the vehicles. Thanks to the law of large numbers, the randomness related to the movement of individual vehicles is washed out by the fluid scaling and the distribution of the travel times concentrates on a single point. This reduces the complexity of the analysis since we are no longer analyzing individual cars but are dealing with a large number of cars which behave in a similar way.

The fluid limit models the regime when the macroscopic area is much larger than the space occupied by a vehicle, and when the number of vehicles in the area is large, which is typically the case during rush hour. The traffic flow can then be thought of as a fluid with smooth dynamics.

Let $\mu(x)$ denote the departure rate in the fluid regime, that is,

$$
\mu(x)=\lim _{N \rightarrow \infty} \frac{\mu_{\lceil N x\rceil}}{N} .
$$

Then, by writing the evolution of $Q(t)$ as the difference of randomly time-changed Poisson processes and applying the functional strong law of large numbers for Poisson processes, we find that the limiting process $q(t)$ adheres the following ordinary differential equation:

$$
q^{\prime}(t)=\lambda(t)-\mu(q(t)) .
$$

In the limiting regime, there is no uncertainty regarding the number of vehicles in the system over time. Setting $w_{n}(\tau, t)=w_{n-1}(\tau, t)=w_{n+1}(\tau, t)$ and $n=q(t)$ in (1), we obtain

$$
\frac{\partial}{\partial t} w(\tau, t)-\frac{\partial}{\partial \tau} w(\tau, t)=-w(\tau, t) \frac{\mu(q(\tau))}{q(\tau)} .
$$

Solving for $w(\tau, t)$ yields

$$
w(\tau, t)=e^{-\int_{\tau}^{t+\tau} \frac{\mu(q(u))}{q(u)} d u}=\frac{e^{-\int_{0}^{t+\tau} \frac{\mu(q(u))}{q(u)} d u}}{e^{-\int_{0}^{\tau} \frac{\mu(q(u))}{q(u)} d u}} .
$$

The mean travel time of a vehicle arriving at time $\tau$ can be computed by integrating $w(\tau, t)$ over $t$, that is,

$$
\bar{w}(\tau)=\int_{0}^{\infty} w(\tau, t) d t .
$$

Substituting from (2) into (3) and using the fact that $-\frac{\partial}{\partial t} w(\tau, t)$ is a probability density function whose integral is equal to 1 for all values of $\tau$, we obtain the following differential equation for the mean travel time:

$$
\frac{d}{d \tau} \bar{w}(\tau)=\bar{w}(\tau) \frac{\mu(q(\tau))}{q(\tau)}-1
$$

that has the solution

$$
\bar{w}(\tau)=\bar{w}(0) e^{\int_{0}^{\tau} \frac{\mu(q(u))}{q(u)} d u}-\int_{0}^{\tau} e^{\int_{s}^{\tau} \frac{\mu(q(u))}{q(u)} d u} d s,
$$

where

$$
\bar{w}(0)=\int_{0}^{\infty} e^{-\int_{0}^{t} \frac{\mu(q(u))}{q(u)} d u} d t .
$$

The above calculations can be used to predict the travel times, given the arrival rate curve and the macroscopic fundamental diagram. To illustrate the approach, Fig. 3 shows three different arrival curves $\lambda(t)$, the corresponding occupancy $q(t)$ and travel time $\bar{w}(t)$ on different panes. The MFD of Yokohama (see Fig. 11 was fitted on a function of the form $\mu(q)=a q e^{b q^{2}+c q+d}$ and this smooth 
function was used for all the calculations. The arrival curves were chosen such that the occupancy reached the range $20-60 \%$ during rush hour, which corresponds to the most characteristic part of the MFD.

The total number of vehicles is the same for all arrival curves, the arrival curves only differing in how concentrated the vehicles arrive during rush hour. These results clearly demonstrate that travel times can significantly be reduced if rush hour is slightly stretched.
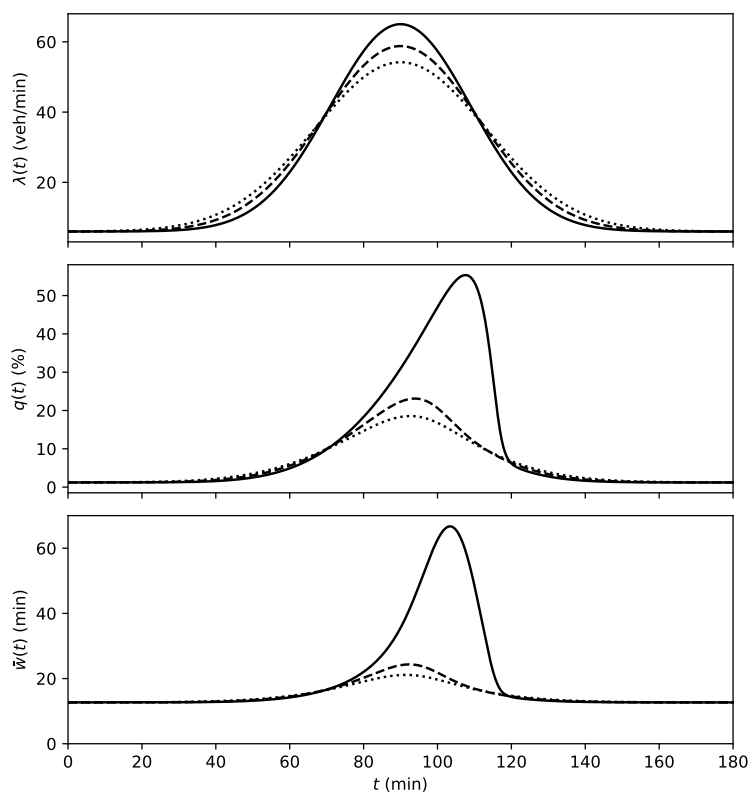

Figure 3: Arrival intensity $\lambda(t)$, mean occupancy $q(t)$ and mean travel time $\bar{w}(t)$ for different arrival intensity curves, and for the Yokohama fundamental diagram.

Using the same arrival curves, Fig. 4 shows $\lambda(t)$ as a function of $q(t)$. The curves are traversed clockwise for increasing values of $t$. At a given value of $q$, the derivative of $q^{\prime}(t)$ is much more negative when $\lambda(t)$ is on the way down. Hence, $q$ decreases more rapidly for the same change in $\lambda$, which results in a flatter lower part.

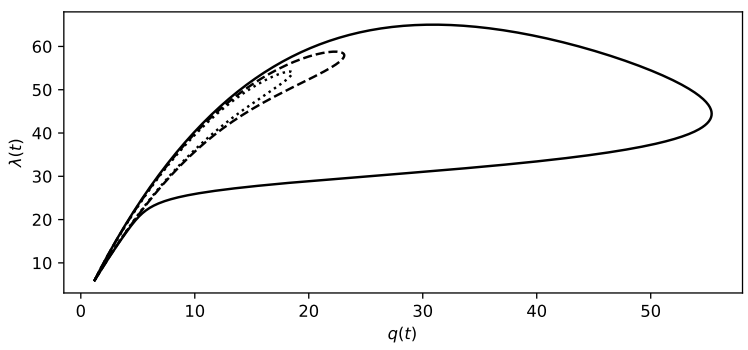

Figure 4: $\lambda(t)$ vs. $q(t)$ (increasing $t$ corresponds to clockwise motion along the curve

\section{Traffic game}

Other than the estimation of travel times of vehicles, (5) and (6) can also be used for dimensioning the capacity of public transport systems. Consider a scenario in which users have access to travel 
time estimates both for commuting by personal vehicles as well as by public transport. If the public transport system (PTS) offers a faster ride, a certain number of users may switch from personal vehicles to the PTS. Assuming users are rational, that is, they choose the mode of transport that minimises their travel times, we use concepts from game theory to compute the number (or the fraction) of users that will switch to the PTS. This can be used to estimate the load on the PTS and dimension its capacity accordingly.

For simplicity, assume that taking the PTS incurs a cost, which may depend upon the itinerary and the time of departure but not upon the congestion in the road network. This cost includes the travel time in the public transport system, the travel time to the access points of the public transport system, the inconvenience of using public transport, costs related to possible disruptions of the service, etc. We assume that this cost can be expressed in terms of travel times: the cost of the public transportation system is the maximal travel time one is willing to undergo.

Note that the assumption that the travel cost does not depend on congestion is natural in case the public transportation system has dedicated lines. This can be either completely separated from the road network like a metro network, or consist of separate lanes embedded in the road network.

\subsection{User equilibrium}

We first investigate the choice made by rational users. When the influence of the choice of one user has negligible impact on the travel times of the population, the solution of the game was proposed by Wardrop 60] in the context of choosing routes in road traffic networks. Known as the Wardrop equilibrium, it says that at the equilibrium choice of routes is such that the cost on each used route is the same and the cost on the unused ones is larger than that of the used ones. We shall apply the concept of Wardrop equilibrium to our time-dependent system to compute the fraction of users who switch to the PTS.

Let $p(t)$ denote the fraction of users that prefer their vehicles over public transport, such that the arrival traffic equals

$$
\lambda(t ; p)=\lambda(t) p(t) .
$$

Moreover, let $\bar{w}(t ; p)$ be the travel time of users arriving at time $t$ that do not choose public transport. Following the arguments of the preceding section, we have

$$
\begin{aligned}
q^{\prime}(t ; p) & =\lambda(t ; p)-\mu(q(t ; p)), \\
\bar{w}(\tau ; p) & =\int_{0}^{\infty} \frac{e^{-\int_{0}^{t+\tau} \frac{\mu(q(u ; p))}{q(u ; p)} d u}}{e^{-\int_{0}^{\tau} \frac{\mu(q u ; p))}{q(u ; p)} d u}} d t .
\end{aligned}
$$

For the computation of $p$, we use the same argument as that for a Wardrop equilibrium. For a given $p$, whenever $\bar{w}(t ; p)<C(t)$, vehicles arriving at time $t$ are better off not using the public transportation system. Hence, unless $p(t)=1$, rational behaviour requires that more users opt for private transportation. Similarly, when $\bar{w}(t ; p)>C(t)$, rationality requires that more users opt for public transportation. Summarising, the optimal proportion of users that choose private transportation, $p^{\circ}(t)$, should satisfy the following constraints:

$$
\begin{cases}\bar{w}\left(t ; p^{\circ}\right)<C(t) & \text { for } p^{\circ}(t)=1, \\ \bar{w}\left(t ; p^{\circ}\right)=C(t) & \text { for } 0<p^{\circ}(t)<1, \\ \bar{w}\left(t ; p^{\circ}\right)>C(t) & \text { for } p^{\circ}(t)=0 .\end{cases}
$$

This means that at the user equilibrium, a fraction $p^{\circ}(t)$ of users will use private transportation whereas $1-p^{\circ}(t)$ will use the PTS.

The function $p^{\circ}$ has no nice and easy analytical solution so we resort to the following iterative numerical algorithm for its computation. Given the arrival curve $\lambda(t)$ and the fundamental diagram $\mu(x)$, we set $p(t)=1$, and then update it according to

$$
p(t) \leftarrow \min \left(1, p(t) e^{-\alpha(\omega(t ; p)-C(t))}\right),
$$



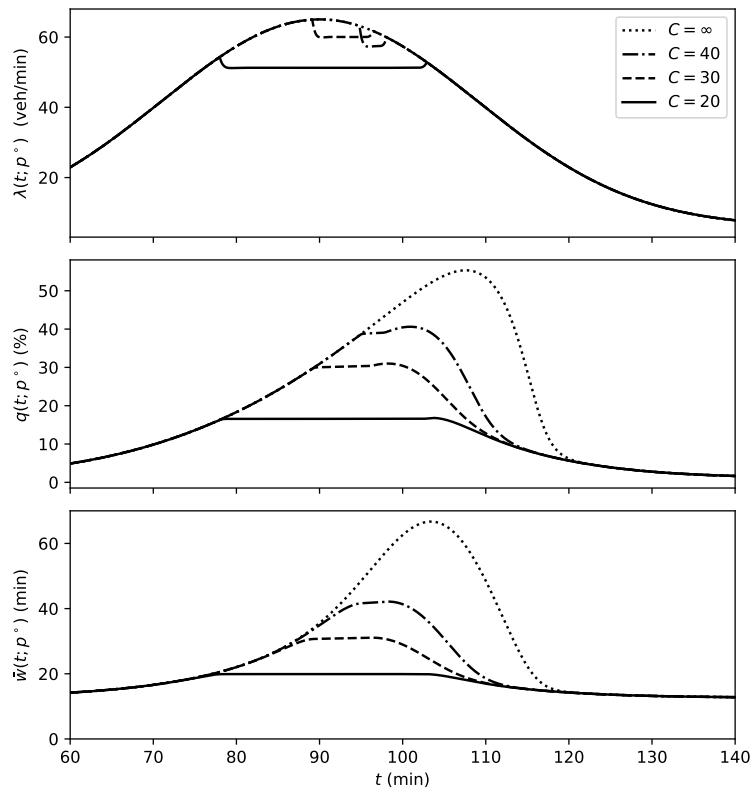

Figure 5: Rational arrival intensity $\lambda\left(t ; p^{\circ}\right)$, mean occupancy $q\left(t ; p^{\circ}\right)$ and mean travel time $\bar{w}\left(t ; p^{\circ}\right)$ for different public transport costs, and for the Yokohama fundamental diagram.

such that $p \rightarrow p^{\circ}$. Here $\alpha$ is a parameter which determines the speed of convergence of the recursion. Small $\alpha$ corresponds to conservative updates, such that convergence is slow. In contrast, for large $\alpha$, the update rule may never converge.

To illustrate the approach, Fig. 5 shows the rational traffic arrival curves $\lambda\left(t ; p^{*}\right)$, the corresponding occupancy $q\left(t ; p^{*}\right)$ and travel time $\bar{w}\left(t ; p^{*}\right)$ on different panes for different values of the cost $C$ as indicated. Note that for $C=\infty$ the PTS is not used, i.e. these curves represent the case that there is no PTS (and no game to be played). The arrival curve (before the game) corresponds to the solid arrival curve from Fig. 3, and the MFD of Yokohama (see Fig. 1) is again used for all calculations. One easily verifies that the travel times indeed do not exceed the fixed cost $C$ of the public transport. Depending on the cost, the PTS is a viable option from the start of the rush hour $(C=20)$, from the peak of the rush hour $(C=30)$ or at the end of the rush hour $(C=40)$. Even when the PTS cost is high and only few people use the PTS, the impact on travel times is considerable. This is in line with the observations of Fig. 3, which showed that a small shift in traffic has a huge impact on travel times.

\subsection{Social optimum and Price of Anarchy}

To benchmark the cost of free transportation choice, we also calculate the socially optimal traffic mix. The total transportation cost $K(t)$ at time $t$, assuming policy $p$ equals,

$$
K(t ; p)=\bar{w}(t ; p) p(t)+(1-p(t)) C(t) .
$$

The socially optimal policy is then given by,

$$
p^{*}(t)=\arg \inf _{p} \int_{T_{1}}^{T_{2}} \lambda(t) K(t ; p) d t
$$

where $\left[T_{1}, T_{2}\right]$ is the rush hour interval. The computation of the social optimum is not exactly an optimal control problem as one would guess at first. The difference is that $\bar{w}$ depends upon the 

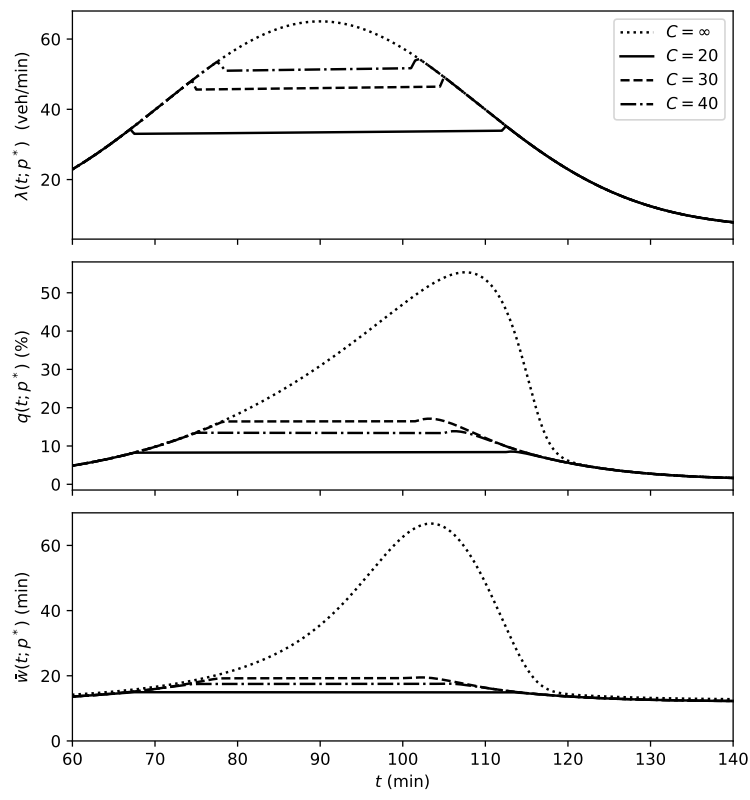

Figure 6: Socially optimal arrival intensity $\lambda\left(t ; p^{*}\right)$, mean occupancy $q\left(t ; p^{*}\right)$ and mean travel time $\bar{w}\left(t ; p^{*}\right)$ for different public transport costs as indicated, and for the Yokohama fundamental diagram.

whole trajectory of $q$ through $\bar{w}(0)$ (see $(6)$ ). This makes it difficult to define the dynamics and hence the Hamiltonian locally as is done in a classical optimal control problem formulation. Define $x(t)=[q(t), \bar{w}(t)]$. We can then approximate (8) by the solution of following optimal control problem:

$$
\min _{p(t) \in[0,1]} \int_{T_{1}}^{T_{2}} \lambda(t) K(t ; p) d t,
$$

subject to

$$
\begin{aligned}
& \dot{x}(t)=\left[\lambda(t) p(t)-\mu(q(t)), \bar{w}(t) \frac{\mu(q(t))}{q(t)}-1\right], \\
& q(0)=q_{0}, \bar{w}\left(T_{2}\right)=\bar{w}(\infty),
\end{aligned}
$$

where $q_{0}=q(\infty)=\mu^{-1}(\lambda(\infty))$ and $\bar{w}(\infty)=q_{0} / \lambda(\infty)$ are the steady-state values of $q$ and $\bar{w}$ when there is no congestion. The inverse of $\mu$ is the one closer to 0 to reflect the lack of congestion. The constraint on $\bar{w}$ is taken on its final value to reduce the influence of the whole trajectory of $q$ on this constraint. We solved (8) using an algorithm similar to gradient descent where the function $p(t)$ is perturbed and the new function is accepted if it yields a lower social cost. The approximate problem (9) was solved using BOCOP which is a freely available solver [61].

Both algorithms gave similar results. From the optimal control formulation, it can be seen that if $\lambda(t)$ goes above a certain value, $\lambda\left(t ; p^{*}\right)$ becomes constant, the value of this constant being equal to the solution of (8) when $q(t)$ does not depend on $t$. More precisely, for fixed cost $C(t)=C$, the constant is equal to $\mu(q)$ where $q$ is the positive fixed point (which exists and is unique for the MFD at hand) of the functional equation,

$$
q=\mu(q) C .
$$

Recall that $p^{\circ}(t)$ is the solution of the set of equations (7). A measure of inefficiency of individual decision making is the Price of Anarchy 62 which is the ratio of the cost at the game equilibrium to 
the optimal social cost,

$$
\mathrm{PoA}=\frac{\int_{T_{1}}^{T_{2}} \lambda(t) K\left(t ; p^{\circ}\right) d t}{\int_{T_{1}}^{T_{2}} \lambda(t) K\left(t ; p^{*}\right) d t} .
$$

The socially optimal arrival curves $\lambda\left(t, p^{*}\right)$ and the corresponding occupancy $q\left(t, p^{*}\right)$ and travel times $\bar{w}\left(t, p^{*}\right)$ are depicted in Fig. 6. We use the same parameters as in Fig. 5. so that we can compare the rational and socially optimal solutions. It is readily observed that a considerably larger fraction of the traffic switches to the PTS in the socially optimal solution, both when the PTS cost is low $(C=20)$ and high $(C=40)$. To calculate the price of anarchy, we consider the cost in the interval $[60,150]$ minutes. We find a $23.2 \%, 35.2 \%$ and $46.8 \%$ increase of the cost for $C=20, C=30$ and $C=40$, respectively.

\section{Conclusion}

We have proposed a queueing-theory-based model for computing the time-dependent travel times during rush hour in a congested area. In contrast to classical queueing models, we account for the macroscopic fundamental diagram of traffic flow by including departure rates which depend on the queue content. As the traffic intensity is high during rush hour, we study the fluid limit of the queueing model at hand. Numerical experiments show that the travel times can be reduced significantly by reducing a small fraction of traffic on the roads. Public transport systems can thus be an effective means of reducing congestion in cities. Relying on game theory, we show how our model can be used to assess the impact of public transport systems on congestion and compare the game-theoretical and socially optimal traffic mixes.

As the current work focuses on the fluid limit, only average travel times are accounted for when comparing public and private transport. One can argue that other travel time metrics like the travel time variance or the complete travel time distribution should be accounted for as well. For example, one can think of a scenario where users opt for public transport if the risk/probability of overly long travel times exceeds a certain value. The tools for approximating such metrics (diffusion limits, control of diffusions) are different and considerably more complicated and therefore considered subject for further study.

\section{References}

[1] J. W. Cohen. The Single Server Queue. North Holland, 1982.

[2] N. Vandaele, T. Van Woensel, and A. Verbruggen. A queueing based traffic flow model. Transportation Research Part D: Transport and Environment, 5(2):121-135, 2000.

[3] X. Chen, Z. Li, L. Li, and Q. Shi. A traffic breakdown model based on queueing theory. Networks and Spatial Economics, 14(3):485-504, Dec 2014.

[4] M. A. A. Boon, R. D. van der Mei, and E. M. M. Winands. Applications of polling systems. Surveys in Operations Research and Management Science, 16(2):67-82, 2011.

[5] https://engage.gov.bc.ca/app/uploads/sites/52/2015/12/Traffic-Data-Overview-2015. pdf. [Online; accessed 10-October-2017].

[6] R. Kühne and N. H. Gartner, editors. Transportation Research Circular E-C149 (75 Years of the Fundamental Diagram for Traffic Flow Theory: Greenshields Symposium). Transportation Research Board, 2011.

[7] H. Lehmann. Distribution function properties and the fundamental diagram in kinetic traffic flow theory. Phys. Rev. E, 54:6058-6064, Dec 1996. 
[8] B. Kerner. The Physics of Traffic - Empirical Freeway Pattern Features, Engineering Applications, and Theory. Springer, Berlin, 2004.

[9] A. Seyfried, B. Steffen, W. Klingsch, and M. Boltes. The fundamental diagram of pedestrian movement revisited. Journal of Statistical Mechanics: Theory and Experiment, 2005(10):P10002, 2005.

[10] L. Ambühl, A. Loder, M. Menendez, and K. W. Axhausen. Empirical macroscopic fundamental diagrams: Insights from loop detector and floating car data. In Proc. of 96th Annual Meeting of the Transportation Research Board (TRB 2017), January 2017.

[11] C. F. Daganzo. Urban gridlock: Macroscopic modeling and mitigation approaches. Transportation Research Part B, 41:49-62, 2007.

[12] N. Geroliminis and C. F. Daganzo. Existence of urban-scale macroscopic fundamental diagrams: Some experimental findings. Transportation Research Part B, 42:759-770, 2008.

[13] C. Buisson and C. Ladier. Exploring the impact of homogeneity of traffic measurements on the existence of macroscopic fundamental diagrams. Transportation Research Record, 2124:127-136, 2009.

[14] T. Van Woensel and N. Vandaele. Modeling traffic flows with queueing networks: a review. Asia-Pacific Journal of Operational Research, 24(4):435-461, 2007.

[15] C.F. Daganzo. The cell transmission model - a dynamic representation of highway traffic consistent with the hydrodynamic theory. Transportation Research Part B - Methodological, 28(4):269-287, 1994.

[16] C.F. Daganzo. The cell transmission model 2. network traffic. Transportation Research Part B Methodological, 29(2):79-93, 1995.

[17] A. Sumalee, R. X. Zhong, T. L. Pan, and W. Y. Szeto. Stochastic cell transmission model (SCTM): A stochastic dynamic traffic model for traffic state surveillance and assignment. Transportation Research Part B - Methodological, 45(3):507-533, 2011.

[18] D. Chowdhury, L. Santen, and A. Schadschneider. Statistical physics of vehicular traffic and some related systems. Physics Reports, 329(4):199 - 329, 2000.

[19] D. Helbing. Traffic and related self-driven many-particle systems. Reviews of Modern Physics, 73(4):1067-1141, 2001.

[20] T. Nagatani. The physics of traffic jams. Reports on Progress in Physics, 65(9):1331-1386, 2002.

[21] D. Heidemann. A queueing theory approach to speed-flow-density relationships. In Proc. of the 13th International Symposium on Transportation and Traffic Theory, Lyon, France, 1996.

[22] R. Boel and L. Mihaylova. A compositional stochastic model for real time freeway traffic simulation. Transportation Research Part B, 40:319334, 2006.

[23] M. J. Lighthill and G. B. Whitham. On kinematic waves ii. a theory of traffic flow on long crowded roads. Proceedings of the Royal Society of London A: Mathematical, Physical and Engineering Sciences, 229(1178):317-345, 1955.

[24] M. Leibig. Pattern-formation characteristics of interacting kinematic waves. Phys. Rev. E, 49:184191, Jan 1994.

[25] H. K. Lee, H. W. Lee, and D. Kim. Steady-state solutions of hydrodynamic traffic models. Physical Review E, 69(1, 2), 2004. 
[26] T Nagatani. Modified kdv equation for jamming transition in the continuum models of traffic. Physica A-Statistical Mechanics and its Applications, 261(3-4):599-607, 1998.

[27] R. L. Anderson, R. Herman, and I. Prigogine. On the statistical distribution function theory of traffic flow. Operations Research, 10(2):180-196, 1962.

[28] I. Prigogine and R. Herman. Kinetic theory of vehicular traffic. Elsevier, 1971.

[29] M. L. L. Iannini and R. Dickman. Kinetic theory of vehicular traffic. American Journal of Physics, 84(2):135-145, 2016.

[30] D. Helbing and B. Tilch. Generalized force model of traffic dynamics. Phys. Rev. E, 58:133-138, Jul 1998.

[31] D. Shamoto, A. Tomoeda, R. Nishi, and K. Nishinari. Car-following model with relative-velocity effect and its experimental verification. Phys. Rev. E, 83:046105, Apr 2011.

[32] Douglas A. Kurtze. Development of kinks in car-following models. Phys. Rev. E, 95:032221, Mar 2017.

[33] W.-L. Jin. On the equivalence between continuum and car-following models of traffic flow. Transportation Research Part B: Methodological, 93:543 - 559, 2016.

[34] K. Nagel and M. Schreckenberg. A cellular automaton model for freeway traffic. J. Phys. I France, 2(12):2221-2229, 1992.

[35] S. Maerivoet and B. De Moor. Cellular automata models of road traffic. Physics Reports-Review Section of Physics Letters, 419(1):1-64, 2005.

[36] A. Schadschneider. Cellular automata models of highway traffic. Physica A: Statistical Mechanics and its Applications, 372(1):142 - 150, 2006. Common Trends in Traffic Systems.

[37] A. Balouchi and D. A. Browne. Finite-size effects in the nagel-schreckenberg traffic model. Phys. Rev. E, 93:052302, May 2016.

[38] J. Zeng, X. Yang, Y. Qian, and X. Wei. Research on three-phase traffic flow modeling based on interaction range. Modern Physics Letters B, 31(35), 2017.

[39] B. S. Kerner. Physics of traffic gridlock in a city. Phys. Rev. E, 84:045102, Oct 2011.

[40] G. Orosz, R. E. Wilson, R. Szalai, and G. Stépán. Exciting traffic jams: Nonlinear phenomena behind traffic jam formation on highways. Phys. Rev. E, 80:046205, Oct 2009.

[41] B. Ray and S. N. Bhattacharyya. Formation of density waves in traffic flow through intersecting roads. Phys. Rev. E, 73:036101, Mar 2006.

[42] Y. Yokoya. Dynamics of traffic flow with real-time traffic information. Phys. Rev. E, 69:016121, Jan 2004.

[43] C. Arita, M. E. Foulaadvand, and L. Santen. Signal optimization in urban transport: A totally asymmetric simple exclusion process with traffic lights. Phys. Rev. E, 95:032108, Mar 2017.

[44] M. Treiber and A. Kesting. Traffic Flow Dynamics: Data, Models and Simulation. Springer, Berlin, 2013.

[45] A. Schadschneider, D. Chowdhury, and K. Nishinari. Stochastic Transport in Complex Systems. Elsevier, Amsterdam, 2011. 
[46] H. Youn, M. T. Gastner, and H. Jeong. Price of anarchy in transportation networks: Efficiency and optimality control. Phys. Rev. Lett., 101:128701, Sep 2008.

[47] A. Rose, R. O'Dea, and K. I. Hopcraft. Price of anarchy on heterogeneous traffic-flow networks. Phys. Rev. E, 94:032315, Sep 2016.

[48] A. Yamauchi, J. Tanimoto, A. Hagishima, and H. Sagara. Dilemma game structure observed in traffic flow at a 2-to-1 lane junction. Phys. Rev. E, 79:036104, Mar 2009.

[49] M. Nakata, A. Yamauchi, J. Tanimoto, and A. Hagishima. Dilemma game structure hidden in traffic flow at a bottleneck due to a 2 into 1 lane junction. Physica A: Statistical Mechanics and its Applications, 389(23):5353 - 5361, 2010.

[50] A. A. Sousa, A. Chaves, G. A. Farias, and F. M. Peeters. Braess paradox at the mesoscopic scale. Phys. Rev. B, 88:245417, Dec 2013.

[51] W.S. Vickrey. Congestion theory and transport investment. American Economic Review, 59:251$261,1969$.

[52] X. Ban, J.-S. Pang, H. X. Liu, and R. Ma. Continuous-time point-queue models in dynamic network loading. Transportation Research Part B: Methodological, 46(3):360 - 380, 2012.

[53] W.-L. Jin. Point queue models: A unified approach. Transportation Research Part B: Methodological, $77: 1-16,2015$.

[54] J. Li and H. M. Zhang. Bounding tandem queuing system performance with variational theory. Transportation Research Part B: Methodological, 81:848 - 862, 2015. ISTTT 21 for the year 2015.

[55] G. F. Newell. Traffic flow for the morning commute. Transportation Science, 22(1):47-58, 1988.

[56] R. Arnott, A. de Palma, and R. Lindsey. Departure time and route choice for the morning commute. Transportation Research Part B: Methodological, 24(3):209 - 228, 1990.

[57] E. J. Gonzales and C. F. Daganzo. Morning commute with competing modes and distributed demand: User equilibrium, system optimum, and pricing. Transportation Research Part B: Methodological, 46(10):1519 - 1534, 2012.

[58] R. Ma and H. M. Zhang. The morning commute problem with ridesharing and dynamic parking charges. Transportation Research Part B: Methodological, 106:345 - 374, 2017.

[59] N. Geroliminis and J. Sun. Properties of a well-defined macroscopic fundamental diagram for urban traffic. Transportation Research Part B, 45:605-617, 2011.

[60] J. G. Wardrop. Some theoretical aspects of road traffic research. ICE Proceedings: Engineering Divisions, 1(3):325-362, 1952.

[61] Team Commands, Inria Saclay. BOCOP: an open source toolbox for optimal control. http: //bocop.org, 2017.

[62] E. Koutsoupias and C. H. Papadimitriou. Worst-case equilibria. In Proceeding of STACS, 1999. 